\title{
Microstructural characterization of Pu-Zr fuels
}

\author{
Assel Aitkaliyeva ${ }^{1,2^{*}}$, Cynthia A. Papesch ${ }^{1}$ \\ ${ }^{1}$ Idaho National Laboratory, Idaho Falls, USA \\ ${ }^{2}$ Department of Materials Science \& Engineering, University of Florida, Gainesville, USA
}

The development of fuels for minor actinide transmutation systems has numerous technical challenges. The fuel has to behave in benign manner during core off-normal events, maintain integrity to high burnup, has to have ease of operation, low minor actinide fabrication loss under remote handling conditions, and lend itself to low-loss recycling processes. High burn-up, high fissile and fertile density capability, inherent safety characteristics, and favorable thermal response of the metallic fuels make them an excellent candidate for next generation fast reactor applications [1]. Despite the work conducted to this date, further research is needed to develop and certify the fuel for applications in transmutation systems. Complete understanding of the irradiation behavior of Pu-based fuels cannot be achieved without the knowledge of the phases and microstructure of unirradiated fuels.

Phases formed in Pu-based systems have been previously experimentally investigated using thermal analysis techniques, X-ray diffraction (XRD), and wavelength and energy dispersive spectroscopy (EDX/WDX) in scanning electron microscopes (SEM) [2-4]. The issue associated with both XRD and SEM analyses is that both techniques are surface-based. In addition, spatial resolution, magnificationdependent sensitivity of EDX/SEM, and overlap between $\mathrm{U}$ and $\mathrm{Pu}$ peaks in EDX results in questionable quantitative chemical analysis of $\mathrm{Pu}$-based phases. To avoid such uncertainties, detailed structural and chemical composition analysis of phases and microstructure of $\mathrm{Pu}-\mathrm{Zr}$ fuels has been conducted in the transmission electron microscope (TEM). The high spatial resolution of TEM allowed characterization of individual matrix phases. Selected-area electron diffraction (SAED) using transmission electron microscopy provided important space-group information and lattice parameters of the phases formed in $\mathrm{Pu}-\mathrm{Zr}$ system.

Surface of the specimen was initially examined using EDX/WDX techniques in a JEOL JSM 7000F field emission SEM operated at accelerating voltage of $20 \mathrm{kV}$. SEM was used to examine the formed microstructure and determine the location of the phases of interest. To reduce charging in the SEM, a layer of Au coating was applied to the sample surface prior to the insertion into the instruments. Crosssectional specimens for transmission electron microscopy (TEM) were prepared in a FEI QUANTA 3D field emission gun (FEG) dual beam focused ion beam/scanning electron microscope (FIB/SEM) using a lift-out approach. Specimens for transmission electron microscopy were characterized in a FEI Tecnai TF30-FEG STwin TEM operated at $300 \mathrm{kV}$. NETZSCH Differential Scanning Calorimeter/Thermogravimetric Analyzer (DSC/TGA) model STA 409 PC was used for analysis. The fuel specimen and sapphire reference material were placed into yttria lined Pt-Rh crucibles. DSC runs were conducted from ambient temperature to $800^{\circ} \mathrm{C}$ followed by cooling to ambient temperature at a rate of $10^{\circ} \mathrm{C} / \mathrm{min}$. Data collection was done during three consecutive heating and cooling cycles and average results will be provided.

Identification of individual phases was conducted in TEM by comparing experimental selective area electron diffraction (SAED) patterns to simulated patterns from published crystal structures produced using JEMS software. In addition, d spacing of each diffraction pattern was measured and compared 
with the published crystallographic data (ICDD PDF2). Five cross-sectional TEM specimens (each approximately $20 \mu m \times 10 \mu m \times 0.1 \mu m$ in size) were prepared from different locations within each specimen. Since the specimens were prepared using a FIB, Ga beam damage and associated artifacts are anticipated. However, the TEM-based examination did not show Ga ion beam-induced artifacts. Figure 1 shows scanning transmission electron micrograph and corresponding SAED patterns of the phases observed in Pu-10Zr fuel alloy. The vertical lines are FIB-induced artifacts that are associated with curtaining. Different contrast features correspond to different phases formed within the fuel alloy. Other fuel compositions will be discussed in the manuscript and relevant data provided.

\section{References:}

[1] D. E. Burkes, R. E. Fielding, D. L. Porter, J. Nucl. Mater. 392, (2009), 158.

[2] M. Kurata, K. Nakamura, T. Ogata, J. Nucl. Mater. 294, (2001), 123.

[3] K. Nakamura, et al, J. Nucl. Mater. 304, (2002), 63.

[4] Y. H. Sohn, et al, J. Nucl. Mater. 279, (2000), 317.

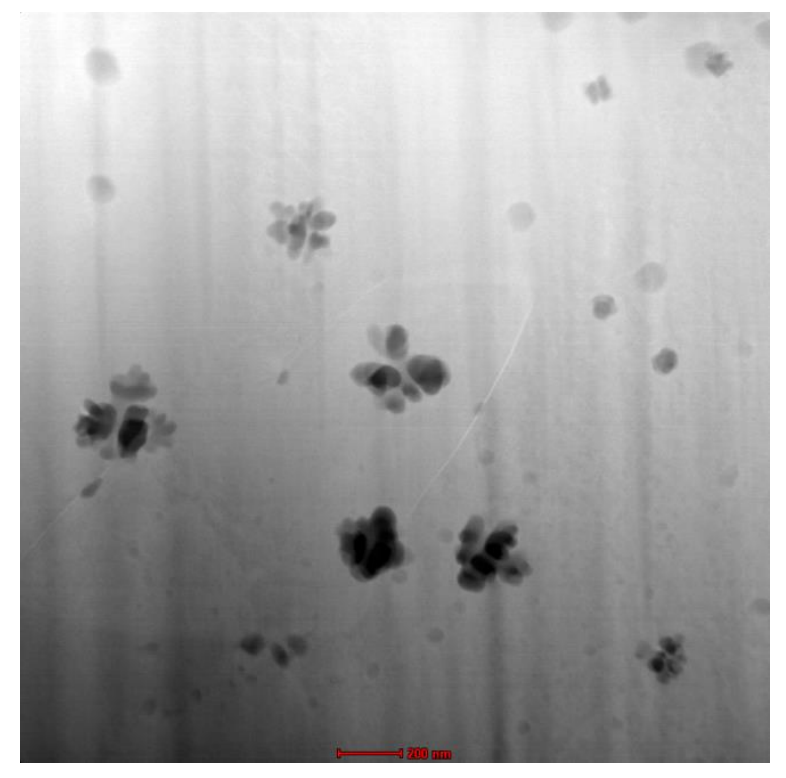

Figure 1. Scanning transmission electron (STEM) micrograph of the microstructure observed in the equilibrated Pu-10Zr alloy. Scale bar denotes $200 \mathrm{~nm}$. 